\title{
HSM2021-2021153 \\ MACHINE LEARNING BASED IDENTIFICATION OF ENERGY EFFICIENCY MEASURES FOR MACHINE TOOLS USING LOAD PROFILES AND MACHINE SPECIFIC META DATA
}

\author{
L. Petruschke ${ }^{1 *}$, G. Elserafi ${ }^{1}$, B. loshchikhes ${ }^{1}$, M. Weigold ${ }^{1}$ \\ ${ }^{1}$ Technical University of Darmstadt, Institute of Production Management, Technology and Machine Tools (PTW), \\ Otto-Berndt-Str. 2, 64287 Darmstadt, Germany \\ ${ }^{*}$ Corresponding author; e-mail: I.petruschke@ptw.tu-darmstadt.de
}

\begin{abstract}
Approaches to detect energy efficiency measures are associated with time consuming analysis requiring expertise. Against this background, this paper presents an expert system to identify potentials for improving the energy efficiency of metal cutting machine tools based on measurement and meta data of 35 machines. For this purpose, it is necessary to determine energy states of machine tools and control strategies of their support units. Therefore, unsupervised and supervised learning algorithms are applied and evaluated. Based on energy states, control strategies and descriptive statistics, performance indicators are developed for enabling automatic selection and prioritization of application-dependent efficiency measures.
\end{abstract}

\section{Keywords:}

machine tool; energy efficiency measures; energy states; expert system

\section{INTRODUCTION}

Due to rising energy prices, legislative pressure, and increasing environmental awareness, energy efficiency in manufacturing is gaining importance [Bunse 2011]. Cutting machine tools represent a share of 1 to $3 \%$ or 200 to 700 TWh of the world's electrical energy demand, which is in the range of Germany's electrical energy demand in 2016 according to [Denkena 2020; Umweltbundesamt 2021].

Machine tools are a reasonable starting point for improving energy efficiency in the manufacturing industry, as they often have high energy losses and hold a significant energy saving potential [Denkena 2020]. Therefore, machine tool operators are faced with the challenge of taking measures to minimize the energy demand of their production machines. Approaches to identify energy efficiency measures are associated with time-consuming measurement data analysis or simulations requiring expertise [Denkena 2020; Petruschke 2020]. Due to insufficient assessment of the economic potential, market failures, and organizational barriers, a discrepancy between the achievable and the actual energy efficiency level, which is called the energy efficiency gap, occurs [Jaffe 1994]. In the case of machine tools, there is also a discrepancy between an increasing availability of energy efficient technologies on the one hand and implemented measures in the industry on the other hand [Denkena 2020; Petruschke 2020]. [Posselt 2016] clusters barriers to the implementation of energy efficiency measures in all sectors into the five categories transparency, priority, economy, capacity, and fragmentation. These barriers could result in lack of knowledge regarding possible energy efficiency measures, undervaluation of a measure's potential or its limited implementation due to deficient process know-how [Sorrell 2000; IEA 2012; Posselt 2016].

At the same time, advances in computer technology, the increasing amount of available data, and the growing opportunities opened by machine learning turn machine learning into a highly economically, socially, and strategically relevant topic [Döbel 2018].

Against this background, this paper presents the expert system, ETA-ExSys, which identifies potentials for improving the energy efficiency of metal cutting machine tools in operation and provides the user with a list of prioritized measures including a monetary assessment. It is developed based on the Cross Industry Standard Process for Data Mining (CRISP-DM) and uses electrical load profiles as well as meta data of metal cutting machine tools as input parameters. Following the introduction, the paper is structured according to CRISP-DM, which consists of six phases, in which we explain the particularly relevant process steps in detail [Wirth 1998]. Section 2 gives an overview of the business and data understanding, followed by data preparation and modeling and evaluation in section 3. The deployment of the developed models in ETA-ExSys and a subsequent discussion follow in section 4. Finally, a summary and conclusion are provided in section 5 .

\section{BUSINESS AND DATA UNDERSTANDING}

The first step in CRISP-DM is the business understanding, in which we describe the initial situation for our research work. This is followed by an explanation of the available database in the data understanding phase. 


\subsection{Business Understanding}

To derive energy efficiency measures from measurement and meta data, it is necessary to determine the energy states of machine tools and control strategies of support units such as cutting fluid supply or machine cooling systems. This analysis has so far been done manually. The required measurement data can be obtained from shortterm mobile measurements, as demonstrated in [Petruschke 2020], whereas meta data can be collected from technical data sheets, type plates, or by interviewing machine operators.

The energy demand of the machine tool, which is required for the subsequent estimation of the savings potential, is determined via the average active power in the respective energy state and its corresponding time share. So far, the different energy states have been identified by defining static threshold values in order to determine their respective time shares. [Petruschke 2020; Petruschke 2021] Various approaches can be found in the literature for analyzing load profiles to identify the energy states. [Petruschke 2021] provide an overview of approaches for identifying energy states on machine and factory level. In addition to the level of detail of the breakdown of energy states, a distinction is also made according to the degree of automation found in the respective approach. [Petruschke 2021]

In literature, a variety of terms and definitions are used for the different energy states of machine tools [Denkena 2020]. The energy states are determined by the states of the main and support unit switches [ISO 2017]. When both the main and support units, in the following referred to as components of a machine tool, are switched on and workpieces are produced within the specified tolerance and cycle time, this energy state is called working [VDMA 2019] or processing [ISO 2017]. The energy state operational is characterized by a higher active power than in the standby state and a lower active power than in working. No workpieces are processed during the states standby and operational. The operational state is often unplanned due to irregular events, e.g., a longer waiting time for the workpiece to be machined. The machine tool can change with minimum time delay between the two energy states operational and working. In standby, the main switch of the machine tool is typically on and spindles and axes are taken out of the control circuit. In addition, support units are partially in switched off. In the energy state off, the main switch as well as the main and support units are switched off. Transitioning between different energy states constitutes a transition state. Powering up, for example, describes the transition from energy state standby to working. [ISO 2017; VDMA 2019] In this paper, we consider the energy states standby, operational and working. The energy state off is only considered in the context of organizational energy efficiency measures, as it does not provide any added value regarding the energy efficiency of components.

To increase energy efficiency, both component-based and organizational measures can be implemented on machine tools. The reduction of non-value adding energy states of the entire machine tool such as standby or operational state is, e.g., an organizational measure [Eberspächer 2017]. An example of a component-based measure is optimizing the nominal power of a low-pressure cooling lubricant pump to avoid overdimensioning [ISO 2017].

In addition to the energy demand of the machine tool and its individual components in the various energy states, the prevailing control strategies of the respective components are also decisive for the evaluation of the savings potential. Different control strategies are applied to pumps, fans, and refrigeration systems for example. These different control strategies in turn result in different load profile characteristics, which according to [Kuhrke 2011; Denkena 2020] are divided into stationary and non-stationary profiles. Within the framework of the developed approach, we distinguish three forms of load profiles: stationary, referred to as constant load profiles as well as nonstationary ones, which are subdivided into discrete and continuous load profiles. In most cases, support units with a fixed speed have constant load profiles that are independent of the operating state. Such units can include, among others, oil extraction units, chip conveyor systems and cooling and lubrication units. An example of a discrete load profile is a cooling lubricant pump that has only one operating point and is switched on and off. The main spindle and drives as well as variable speed pumps have a continuous load profile, e.g., due to acceleration and deceleration processes. [Kuhrke 2011; Denkena 2020] To identify the predominant control strategy of the respective component, load curves and load duration curves of the main power supply and the respective components have been manually analyzed so far [Petruschke 2020].

The respective measure can be evaluated based on the determined energy demand and control strategy. The savings potential is calculated using empirical values while taking meta data into account. Therefore, the selection of suitable measures depends on the knowledge and experience of the person carrying out the analysis. Selected measures are prioritized based on their expected respective costs and savings potential.

Since the process of selecting and prioritizing energy efficiency measures is time-consuming and often requires expert knowledge, it poses a challenge for businesses operating machine tools. This challenge involves the maintenance and energy management departments in particular. Therefore, the presented expert system aims to contribute to reducing barriers to the implementation of measures by shortening, simplifying, and automating the process. The system boundary of the considered system is defined according to [Gontarz 2015; ISO 2017; ISO 2018] so that the machine tool is supplied with electrical energy and compressed air from outside the system boundary, as is often the case in practice.

\subsection{Data Understanding}

In the following, we start by covering the data understanding of the available data. To automatically determine energy states of machine tools and control strategies of support units, we apply and evaluate unsupervised as well as supervised learning algorithms.

This is done using measurement data of 35 metal cutting machine tools with different configurations and production environments, obtained in the research project ETATransfer. For each machine tool, the database contains active power data points of the main power supply and up to six other components, recorded at a sampling rate of $1 \mathrm{~Hz}$. A total of 4,300 hours of multivariate time series were recorded. Of these, 2,471 hours were labeled with energy states and 90 univariate time series with a total length of 8,653 hours resulting from the support units were labeled with control strategies. The data were labeled manually by experts. In terms of the entire database, the energy state working has the largest time share with slightly more than $60 \%$. The energy states operational and standby are represented at approximately $20 \%$ each. [Petruschke 2021] However, not all machine tools necessarily include all three energy states and their measurement periods may vary in length. As with the energy states, univariate time series were labeled manually with control strategies based 
on the load profiles. Of the 90 labeled time series, 19 exhibit a constant load profile, 58 a discrete load profile and 13 a continuous load profile. In addition, nominal power, year of manufacture, machine type, shift operation, etc. can be used as meta data for the identification of energy efficiency measures. However, in our approach, we only use the nominal power due to inconsistant data availability.

\section{MODEL DEVELOPMENT AND EVALUATION}

The developed models aim to identify energy states and control strategies using supervised and unsupervised learning algorithms to evaluate their performance. Since the steps data preparation, modeling, and evaluation differ for the two identification tasks and the investigated machine learning approaches, the identification of energy states and control strategies are considered separately in the following sections.

\subsection{Energy States}

Since we consider the three energy states working operational and standby, the task at hand is treated as a multiclass classification problem [Tewari 2005] for which we investigate the unsupervised and supervised learning algorithms listed in table 1. Unsupervised learning algorithms are used to find patterns in unlabeled data in order to classify it into clusters [Doleski 2020]. For these algorithms data processing steps were applied according to the flowchart shown in figure 1. Initially, the measurement data is filtered as stated by [Brownlee 2018] to eliminate outliers and reduce measurement noise. To select the most suitable filter, we investigate the effect of different filters with varying parameters on the performance of different unsupervised algorithms. The investigation compares the median filter, the Wiener filter and the Butterworth filter from the Python library SciPy [Virtanen 2020]. Since no energy state is severely underrepresented, the accuracy as described in [Grandini 2020] was used as a performance metric. For the examined time series, the filter and parameter study results in the highest accuracy when using the median filter with a filter width of 499 seconds.

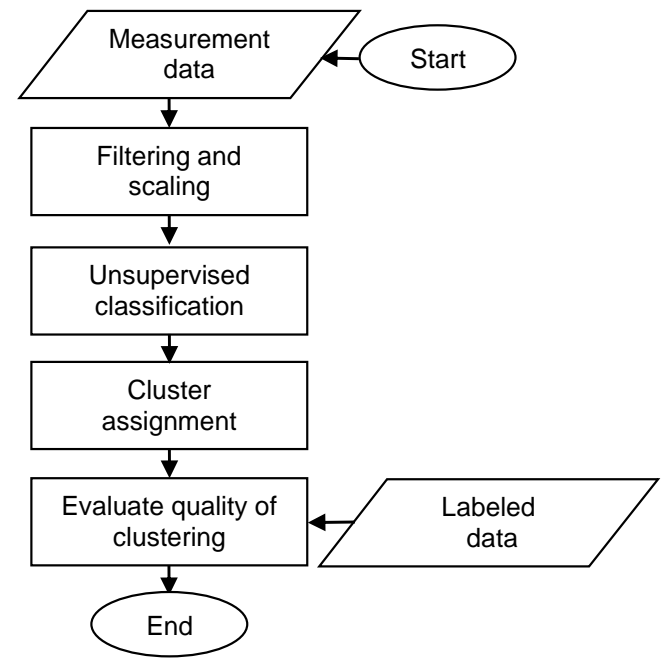

Fig. 1: Flowchart of unsupervised learning.

Within subsequent data preparation, the filtered data is scaled due to the varied range of values. While some machine learning algorithms can only work properly with scaling, it could lead to faster convergence or increased accuracy for other algorithms [Müller 2017]. For scaling multivariate time series, different alternatives from the Python library scikit-learn [Pedregosa 2011] were examined. The best results for the available data in terms of accuracy were achieved by the normalizer, which treats the data of all measured components within one second as a vector and divides it by its norm [Hackeling 2014]. After preprocessing, the unsupervised learning algorithms listed in table 1 are applied to the data of all 35 machine tools using three clusters, one for each energy state. The clusters are assigned to the energy states standby, operational and working according to their average active powers. For evaluation, the average accuracies of the unsupervised learning algorithms are compared and the clustered load profiles are visually inspected, as exemplified in figure 2. After hyperparameter adjustment, the Gaussian Mixture algorithm achieves the best results with an average accuracy of $82.36 \%$ along all 35 machine tools.

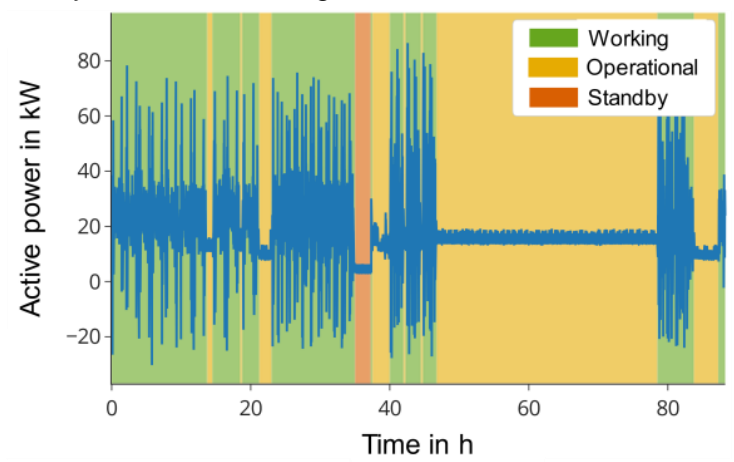

Fig. 2: Exemplary energy state classification using Gaussian Mixture for visual inspection.

Supervised learning uses labeled training datasets containing input as well as output values. This enables the trained models to assign new elements into existing classes [Doleski 2020]. In practice, the dataset is usually divided into a training set and a test set. The training set is used to train classifiers, while the test set contains unseen examples and is used to evaluate and compare the classifiers [Fernandes de Mello 2018]. There are several options for splitting datasets into a training and a test set. In this paper, we use Leave-One-Group-Out cross validation (LOGO CV) implemented in scikit-learn [Pedregosa 2011] for splitting the datasets. The LOGO CV is a modification of cross validation as described in [Kohavi 1995], which divides the data into groups each representing data from one machine tool. With the described database the LOGO CV performs a total of 35 iterations. In each iteration, training is performed with data from 34 machine tools and tested with data from the remaining machine tool, meaning each test run is performed with data from a different machine tool that is not included in the training data. This group splitting is necessary for preventing data leakage between training and test data and hence obtaining a representative performance estimation for unknown machine tools [Kaufman 2011]. Figure 3 shows the group splitting for the first five machine tools of the database (twocolor bars) as well as the distribution of manually labeled energy states (tricolor bar).

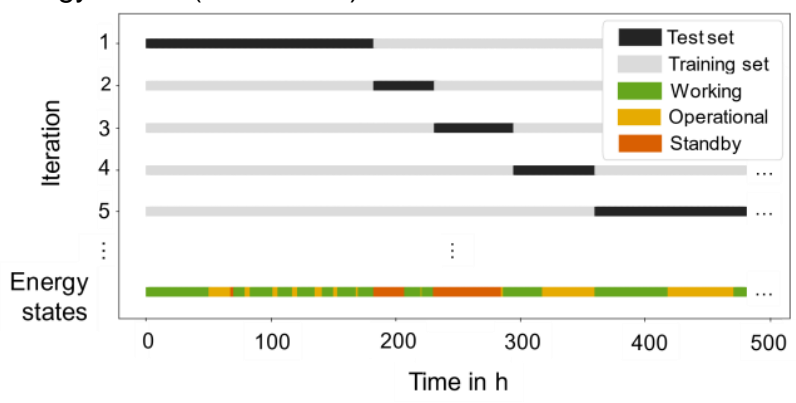

Fig.3: Database insight and iterative model validation process for the identification of energy states. 
As shown in [Brownlee 2018], the results of classifying time series data using supervised learning can be improved by using features based on a fixed window size. Consequently, we used the Time Series Feature Extraction Library (TSFEL) [Barandas 2020] for Python to calculate features and subsequently perform feature selection by deleting features that are not relevant to the target variable using the feature importance [Butz 2006]. Removing unimportant features results in faster and, in some cases, more accurate models [Kuhn 2013]. Furthermore, the window size for feature extraction was varied to find the best window size. Figure 4 shows an overview of the described process.

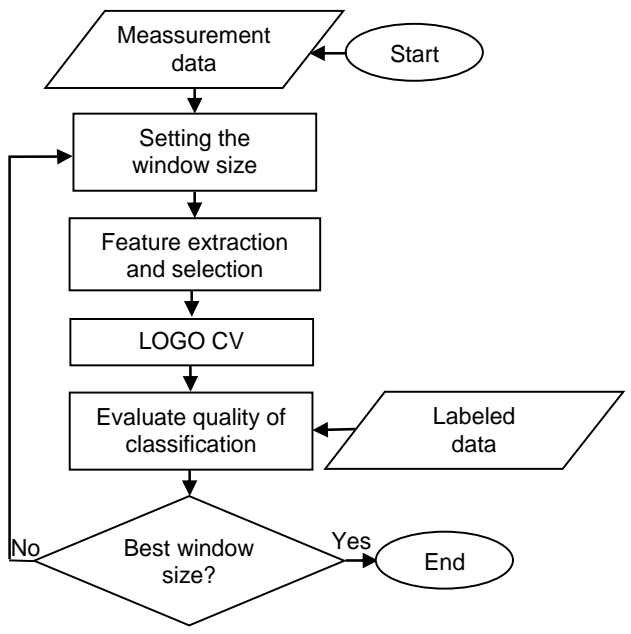

Fig. 4: Flowchart of supervised learning.

After hyperparameter optimization using grid search [Müller 2017], the Random Forest Classifier achieves the highest average accuracy among the supervised learning algorithms with $83.78 \%$ using a window size of 15 minutes. Thus, the accuracy of the Random Forest Classifier is slightly higher than that of the Gaussian Mixture. Moreover, since unsupervised learning methods do not undergo a training process, their accuracy is not affected by the amount of available training data. In contrast, supervised learning methods such as the Random Forest Classifier could potentially achieve a higher accuracy with more training data.

\subsection{Control Strategies}

Since we assign constant, discrete, or continuous load profiles per univariate time series, we only investigate

supervised learning algorithms for identifying the control strategy class. To investigate supervised learning algorithms for this task, we follow a similar approach to the one shown in figure 3. However, due to the small database, additional data is acquired by splitting the time series evenly. Dynamic Time Warping (DTW) is used for automatically ensuring the similarity of the original time series to time series splits [Yang 2019]. Through empirical investigation using the existing database, the following similarity condition is defined:

$|\mathrm{DTW}| \leq 1$

If time series sections meet this condition, they are included in an extended database with the same label as the original time series. Figure 5 shows the exemplary division of a time series into three sections. The first two sections from the left have a similar (continuous) load profile to the original time series according to the upper definition. In contrast, the right section shows a constant load profile and is thus not similar to the original time series. With this approach, the original database of 90 time series can be extended to include 12,332 time series sections. With further division, the quality of the classification starts to decrease because of the more difficult distinction of too short sections.

Due to the unbalanced dataset, the weighted f1-score [Hammerla 2016] is additionally considered as a performance metric. The conducted studies indicate that the splitting of the time series has a beneficial impact on the model quality. After hyperparameter optimization using grid search [Müller 2017], the Support Vector Classifier achieves the best result with an average accuracy of $95.65 \%$ and an average weighted f1-score measure of $95.55 \%$.

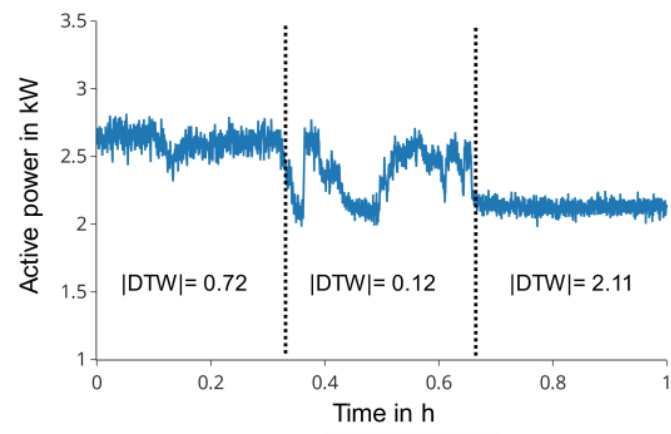

Fig. 5: Time series split with calculated DTW.

\begin{tabular}{cccc}
\hline classification task & learning method & algorithm & average accuracy \\
\hline energy states & unsupervised & k-Means & $75.65 \%$ \\
energy states & unsupervised & k-Shape & $42.31 \%$ \\
energy states & unsupervised & Gaussian Mixture & $82.36 \%$ \\
energy states & supervised & Logistic Regression & $71.85 \%$ \\
energy states & supervised & k-Neighbors Classifier & $76.77 \%$ \\
energy states & supervised & Support Vector Classifier & $79.12 \%$ \\
energy states & supervised & Random Forest Classifier & $83.78 \%$ \\
energy states & supervised & Linear Discriminant Analysis & $79.88 \%$ \\
energy states & supervised & Gaussian Naive Bayes & $60.59 \%$ \\
control strategies & supervised & Logistic Regression & $89.87 \%$ \\
control strategies & supervised & k-Neighbors Classifier & $81.84 \%$ \\
control strategies & supervised & Support Vector Classifier & $95.65 \%$ \\
control strategies & supervised & Random Forest Classifier & $87.32 \%$ \\
control strategies & supervised & Linear Discriminant Analysis & $91.83 \%$ \\
control strategies & supervised & Gaussian Naive Bayes & $92.97 \%$ \\
\hline
\end{tabular}

Tab. 1: Comparison of average accuracy of investigated algorithms for different classification tasks. MM Science Journal | 2021 | NOVEMBER - Special Issue on HSM2021 


\section{DEPLOYMENT AND DISCUSSION}

The presented models for determining energy characteristics are deployed in ETA-ExSys to enable identifying and prioritizing energy efficiency measures. The model results are combined with energy key performance indicators (KPIs) to ensure a transparent recommendation process. The performance of ETA-ExSys is discussed subsequently.

\subsection{ETA-ExSys}

ETA-ExSys aims to assist in decisions regarding energy efficiency measures by providing them with recommended measures and their respective evaluations. The automated identification of suitable measures is based on energy KPIs, which are calculated using results from the preceding data analysis. The measures are selected from a pre-defined list, as will be further explained in the following.

\section{Energy KPIs}

As defined in [VDMA 2010], KPls are employed for measuring progress of important goals or critical success factors. The following work defines a set of energy KPIs for evaluating the analyzed system regarding its energy consumption and identifying suitable energy efficiency measures. The presented energy KPls are derived according to the business and data understanding in addition to existing KPls in [Dehning 2019] and are calculated using statistical analysis of the measurement data.

To provide a holistic evaluation of a given machine tool and identify unit-specific measures, we define energy KPIs on machine, and component levels, as shown in table 2.

The maximum machine degree of utilization $(M M D U)$ is defined as the ratio of the machine's maximum active power $P_{\max }$ to its nominal power $P_{\text {nominal }}$. This conveys information regarding the dimensioning of the machine e.g., a low $M M D U$ could hint at an overdimensioned machine tool.
Relative state-dependent time share $\left(R S T_{S}\right)$ and energy consumption $\left(R S E_{S}\right)$ establish the respective share of an energy state $S$ in the total operation time $t_{\text {total }}$ and energy consumption $E_{\text {total }}$. Both energy KPls help in identifying optimization approaches regarding the energy states during machine operation.

As with $M M D U$, the maximum degree of utilization $\left(M D U_{i}\right)$ of a component $i$ is defined as the ratio of its maximum active power $P_{\max , i}$ to its nominal power $P_{\text {nominal }, i}$ and carries information about its dimensioning.

The relative energy consumption $\left(R E_{i}\right)$ of a machine component $i$ relates its energy consumption $E_{i}$ to the machine's total energy consumption $E_{\text {total }}$. This information assists in finding and prioritizing energy saving potentials for each component.

Combining $R E_{i}$ with $R S T_{S}$ results in the relative component and state-dependent energy consumption $\left(R E S_{i, S}\right)$ of a component $i$ in an energy state $S$, which helps in spotting non-production losses.

Another KPI for determining whether a component $i$ is being operated in a needs-based manner is the drive correlation $\left(D C_{i}\right)$. As the magnitude of the correlation factor $r$ between the component's and the drives' operation within the machine tool, $D C_{i}$ reveals how strongly the component operation is associated with the metal cutting process and can help in detecting non-production losses. The correlation factor $r$ is defined in [Kohn 2011].

Finally, the recuperation factor $R F$ determines whether energy is recuperated from braking processes in the machine's drives. This is implied, when the share of time with negative energy consumption $t_{R}$ exceeds $0.1 \%$ of total operation time $t_{\text {total }}$. In this case, the proportion of recuperated energy $E_{R}$ of the total energy $E_{\text {total }}$ is also calculated. Otherwise $R F$ is equates to 0 .

\begin{tabular}{|c|c|c|c|}
\hline Machine level energy KPIs & Definition & Component level energy KPIs & Definition \\
\hline $\begin{array}{l}M M D U \\
\text { Maximum machine degree of utilization }\end{array}$ & $M M D U=\frac{P_{\text {max }}}{P_{\text {nominal }}}$ & $\begin{array}{l}M D U_{i} \\
\text { Maximum degree of utilization }\end{array}$ & $M D U_{i}=\frac{P_{\text {max }, i}}{P_{\text {nominal }, i}}$ \\
\hline $\begin{array}{l}\text { NPTF } \\
\text { Non-production time factor } \\
\text { NPLF } \\
\text { Non-production load factor }\end{array}$ & [Dehning 2019] & $\begin{array}{l}R E_{i} \\
\text { Relative energy consumption }\end{array}$ & $R E_{i}=\frac{E_{i}}{E_{\text {total }}}$ \\
\hline $\begin{array}{l}N P E F \\
\text { Non-production energy factor } \\
B E S F \\
\text { Benchmark energy saving factor }\end{array}$ & [Dehning 2019] & $\begin{array}{l}R E S_{i, S} \\
\text { Relative component and } \\
\text { state-dependent energy } \\
\text { consumption }\end{array}$ & $R E S_{i, S}=R E_{i} * R S T_{S}$ \\
\hline $\begin{array}{l}\text { PESF } \\
\text { Powering up and down energy saving } \\
\text { factor }\end{array}$ & [Dehning 2019] & $\begin{array}{l}D C_{i} \\
\text { Drive correlation }\end{array}$ & $D C_{i}=|r|$ \\
\hline $\begin{array}{l}R S T_{S} \\
\text { Relative state-dependent time share }\end{array}$ & $R S T_{S}=\frac{t_{S}}{t_{\text {total }}}$ & $R F$ & $0, \frac{t_{R}}{t_{\text {total }}}<0.1 \%$ \\
\hline $\begin{array}{l}R S E_{S} \\
\text { Relative state-dependent energy consun }\end{array}$ & $R S E_{S}=\frac{E_{S}}{E_{\text {total }}}$ & Recuperation factor & $\frac{\left|E_{R}\right|}{E_{\text {total }}}, \frac{t_{R}}{t_{\text {total }}} \geq 0.1 \%$ \\
\hline
\end{tabular}

Tab. 2: Energy KPls on machine and component levels. 


\section{Energy Efficiency Measures}

Energy efficiency measures are selected from a catalogue of measures compiled from [CECIMO 2005], [Eberspächer 2017] and [ISO 2017] and are divided into a componentbased section and an organizational section. Componentbased measures can only be selected when componentspecific measurement data is available. Organizational measures, however, can always be selected, even when only the main power supply had been measured. To be selected, each measure must fulfill a measure condition relating to a specific energy $\mathrm{KPI}$ - this also takes into consideration, whether the measure is already implemented in the system. For component-based measures, however, measurement data must first be available for the respective component.

For comparison, measures are paired with a cost factor $C_{M}$ and an energy saving potential factor $S P_{M}$, which are mostly experience-based, as introduced in [VDI 1998]. Measures are identified according to the calculated energy KPIs as well as the recognized control strategy of the machine tool and ranked depending on their respective priority number $P N$. Measures relating to the control strategy are only recommended when an inefficient control strategy is detected from the measurement data. The componentbased priority number $P N_{i}$ is calculated using the relative energy consumption $R E_{i}$ of the corresponding component, as shown in (2). Component-based measures are selected and prioritized as illustrated in figure 6 .

$P N_{i}=R E_{i} \cdot C_{M} \cdot S P_{M}$

For organizational measures, such as powering down during non-productive times, the priority number $P N_{O}$ is calculated as shown in (3), using in this case the $N P T F$. The range variable $R V_{K P I}$ given in (4) depicts the value of a specific KPI relative to the respective range $K P I_{\max }-K P I_{\min }$ calculated from the dataset, which represents a benchmark from the observed machines.

$P N_{O}=R V_{K P I} \cdot C_{M} \cdot S P_{M}$
$R V_{K P I}=\frac{K P I}{K P I_{\max }-K P I_{\min }}$

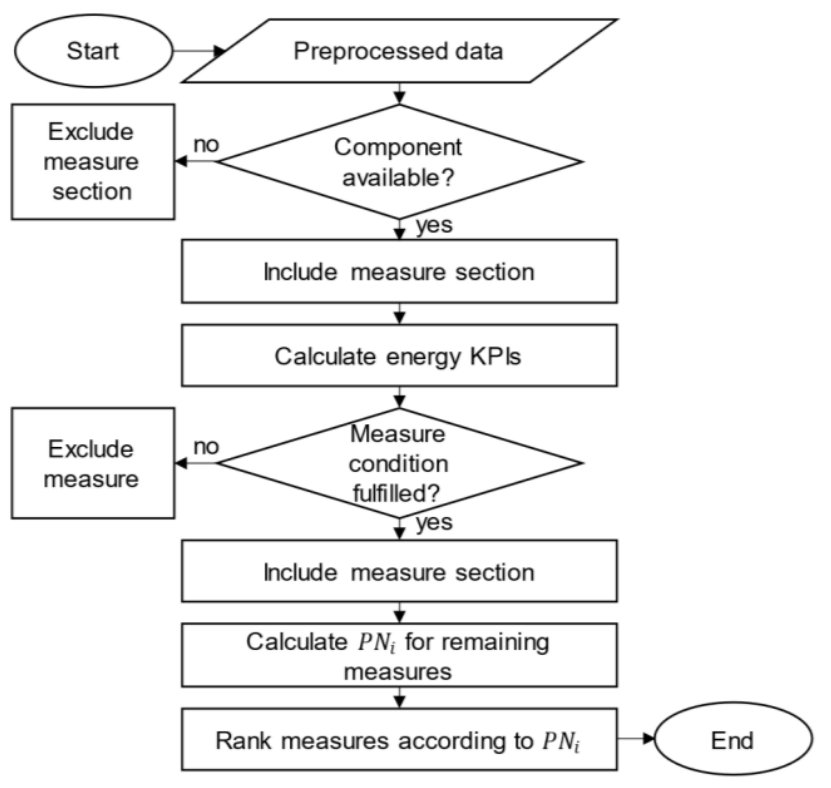

Fig. 6: Flowchart of component-based measure selection and prioritization.

Since it is difficult to generally quantify the monetary effects of component-based measures, we focus on the energy costs of the identified energy states. By multiplying the daily operation time and annual operation days with the relative state-dependent time share $R S T_{S}$, the annual operation time per energy state is determined. Combined with the average active power $P_{\text {avg, } i}$, ETA-ExSys automatically deduces the state-specific energy costs and calculates the energy saving potential of organizational measures such as powering down during non-productive energy states (e.g., operational, or standby).

\section{System Structure}

The following will present functions und processes within the expert system and interactions with the user interface. All data processing and analysis functions as well as the user interface are programmed in Python. The structure of ETA-ExSys as well as interactions between its constituents are illustrated in figure 7.

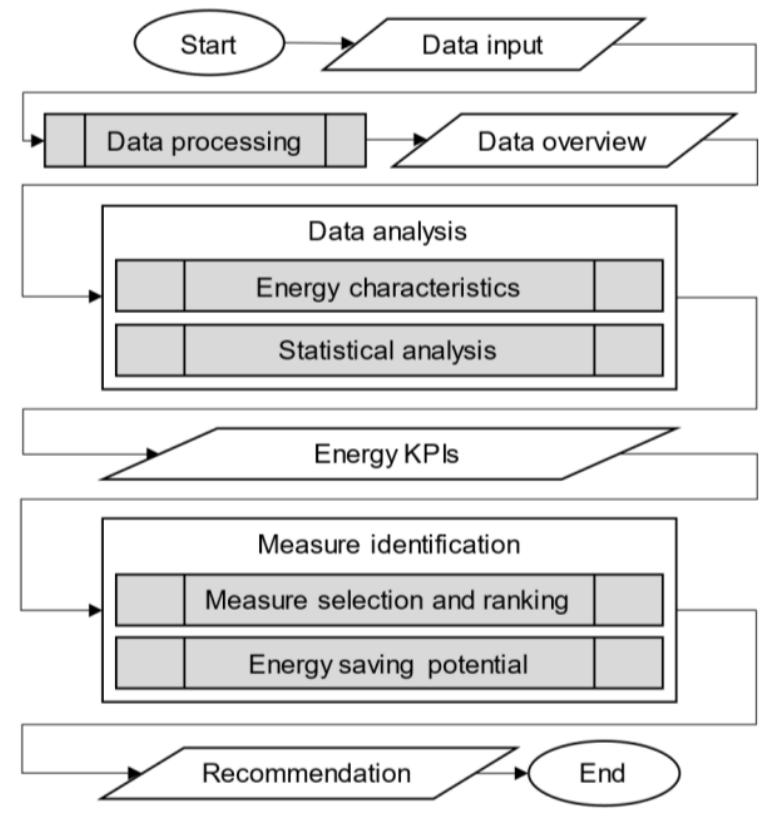

Fig. 7: Structure and functions of ETA-ExSys.

The front end is programmed as a web application, which is organized into four sections: data input, data overview, data analysis and recommended measures. As the name suggests, the data input section allows the user to upload measurement data and provide meta data about the machine tool as well as its operation for a comprehensive description of the system. Following the data upload, data processing takes a few minutes to be completed in the back end, making the three remaining sections accessible to the user.

An overview of the processed data is provided via interactive plots and diagrams in the data overview section. By depicting findings and correlations within the data, the presented overview provides the user with important system insights and maintains transparency in the recommendation process.

The section data analysis gives graphical representations of the identified energy states, resulting from the analysis of energy characteristics in the back end. The different energy state representations supply information about time and energy shares of each energy state as well as state-specific energy consumption of different machine components. This information further aids the user in understanding system recommendations and gaining enough knowledge for their own plausibility check.

The final section, recommended measures, assembles the ranked organizational and component-based measures 
as well as the estimated energy saving potential by adjusting the energy states.

As previously mentioned, the recommended measures are selected from a measure catalogue and ranked according to their priority numbers, which are calculated using results from the back-end statistical analysis of the measurement. This process takes place in the back end block measure identification. The calculated values of the energy KPIs shown in table 2 can be downloaded by the user for further analysis.

\subsection{Discussion}

To validate the expert system, we implement it to an industrial use-case with one machine tool and compare the automatically recommended measures with manually selected ones. The comparison shows that measures selected and ranked by ETA-ExSys appear to be more specific and differentiated as opposed to the more generalized manually selected measures. The expert system's performance is further underlined by the short computation time and high degree of automation in contrast to the lengthy process of manually selecting efficiency measures. This makes the approach suitable for use on numerous other machine tools in existing production sites.

Additionally, the measure ranking feature of the expert system further assists in prioritizing efficiency measures, which is usually lacking in manual selection. Nevertheless, the priority numbers $P N$, which are used for measure ranking, strongly depend on energy saving factors $S P_{M}$ and cost factors $C_{M}$, which are experience-based. Due to discrepancies in $S P_{M}$ and $C_{M}$ over different use-cases, the suggested factors can and should be adjusted by the user if necessary.

On the other hand, the energy saving potential of staterelated organizational measures, gives the user quantitative information about the monetary effects of adjusting energy state time shares for a concrete decision basis.

The developed system considers electrical power as the sole input energy, which shapes the identification of the system's energy characteristics. This case is realistic for some industrial settings, however, several practical usecases with additional energy forms such as compressed air or chilled water are therefore not compatible with the expert system's framework.

Finally, the more diverse training data is available, the higher the chances are to train models with a higher accuracy and better generalization performance. In our case, supervised learning algorithms perform better for the identification of energy states than unsupervised ones. However, the datasets used for supervised learning are manually labeled and therefore do not necessarily represent an absolute truth. Furthermore, multivariate datasets allowed higher accuracies compared with univariate datasets for the identification of energy states. Consequently, adding more correctly labeled data from machine tools with different energy forms and topologies to the training set should significantly increase the system's accuracy and widen its range of application.

\section{SUMMARY AND CONCLUSION}

In accordance with CRISP-DM, a software-based expert system was developed. For this purpose, different supervised and unsupervised machine learning algorithms were compared regarding their suitability for the identification of energy states and control strategies of machine tools in operation. In terms of performance, supervised learning algorithms achieved the best results for the identification of energy characteristics. For the identification of energy states, the Random Forest Classifier showed the highest average accuracy with $83.78 \%$, while the Support Vector Classifier achieved the highest average accuracy of $95.65 \%$ for the identification of control strategies. In addition to the identified energy characteristics, further statistical data are used to determine energy KPIs. Energy efficiency measures are selected based on the calculated energy KPIs and the detected control strategy. The measures are then prioritized depending on their expected energy savings, associated costs, and respective energy KPI values. Assessment indicates that ETA-ExSys succeeds in automatically identifying suitable energy efficiency measures in a significantly shorter time compared to manual analysis and measure selection.

It is expected that an increase in the amount of data will further contribute to the accuracy and generalization performance of the models. With the inclusion of other forms of energy besides electrical energy, ETA-ExSys can be applied to a wider range of use-cases in the future. Moreover, a broader data basis of implemented energy efficiency measures can improve measure prioritization by providing more realistic cost and energy saving factors for calculating the priority number. For future research, investigating models that do not require the intermediate step of calculating energy KPIs, might be promising. This could possibly describe the direct relation between measurement data and proposed energy efficiency measures and reduce the influence of human error in the choice of KPIs. Furthermore, transferring this approach to other machine types, such as cleaning machines, could also potentially uncover future research topics.

\section{ACKNOWLEDGMENTS}

The presented results have been achieved within the research project ETA im Bestand (grant agreement no. 03EN2048A) which is funded by the Federal Ministry for Economic Affairs and Energy (BMWi) and managed by the management agency Project Management Jülich (PtJ).

The work is based on measurement data obtained in the research project ETA-Transfer (Grant Agreement No. 46ETA01) which is funded by the Federal Ministry for Economic Affairs and Energy (BMWi) and managed by the Federal Office for Economic Affairs and Export Control (BAFA)

\section{REFERENCES}

[Barandas 2020] Barandas, M., et al. TSFEL: Time Series Feature Extraction Library. SoftwareX, 2020, 11, 100456, 10.1016/j.softx.2020.100456

[Brownlee 2018] Brownlee, J., Ed. Deep Learning for Time Series Forecasting: Predict the Future with MLPs, CNNs and LSTMs in Python, 2018.

[Bunse 2011] Bunse, K., et al. Integrating energy efficiency performance in production management gap analysis between industrial needs and scientific literature. Journal of Cleaner Production, 2011, 19, 6-7, 667-679, 10.1016/j.jclepro.2010.11.011.

[Butz 2006] Butz, M.V. Rule-based evolutionary online learning systems. A principled approach to LCS analysis and design. Studies in fuzziness and soft computing 191, Berlin: Springer, 2006, 3540253793. [CECIMO 2005] CECIMO. Roadmap for CECIMO's SelfRegulative Initiative (SRI) for the Sector specific 
implementation of the Directive 2005/32/EC (EuP Directive) for 2009 to 2011, Brussels, 2005.

[Dehning 2019] Dehning, P., et al. Load profile analysis for reducing energy demands of production systems in non-production times. Applied Energy, 2019, 237, 117130, 10.1016/j.apenergy.2019.01.047.

[Denkena 2020] Denkena, B., et al. Energy efficient machine tools. CIRP Annals, 2020, 69, 2, 646-667, 10.1016/j.cirp.2020.05.008.

[Döbel 2018] Döbel, I., et al. Maschinelles Lernen. Eine Analyse zu Kompetenzen, Forschung und Anwendung, München: Fraunhofer-Gesellschaft zur Förderung der angewandten Forschung e.V., 2018.

[Doleski 2020] Doleski, O.D. Realisierung Utility 4.0 Band 2, Wiesbaden: Springer Fachmedien Wiesbaden, 2020, 978-3-658-25588-6.

[Eberspächer 2017] Eberspächer, P. Zustandsmodellbasierte, steuerungsnahe Energieverbrauchsoptimierung von Werkzeugmaschinen. Dissertation, Universität Stuttgart, 2017.

[Fernandes de Mello 2018] Fernandes de Mello, R., and Antonelli Ponti, M. Machine Learning, Cham: Springer International Publishing, 2018, 978-3-319-94988-8.

[Gontarz 2015] Gontarz, A.M. Energy Assessment of Machine Tools within Manufacturing Environments. Dissertation, ETH Zürich, 2015.

[Grandini 2020] Grandini, M., et al. Metrics for Multi-Class Classification: an Overview, 2020.

[Hackeling 2014] Hackeling, and Gavin. Mastering machine learning with scikit-learn, 2014.

[Hammerla 2016] Hammerla, N.Y., et al. Deep, Convolutional, and Recurrent Models for Human Activity Recognition using Wearables, 2016.

[IEA 2012] IEA. Key world energy statistics 2012. International Energy Agency: OECD Publishing, 2012.

[ISO 2017] ISO. International Organization for Standardization. Machine tools - Environmental evaluation of machine tools. Part 1: Design methodology for energy-efficient machine tools, Geneva, 2017, ISO 14955-1.

[ISO 2018] ISO. International Organization for Standardization. Machine tools - Environmental Evaluation of Machine Tools. Part 2: Methods for measuring energy supplied to machine tools and machine tool components, Geneva, 2018, 14955-2.

[Jaffe 1994] Jaffe, A., and Stavins, R.N. The EnergyEfficiency Gap: What Does It Mean? Energy Policy, 1994, 1994, 22, 804-810.

[Kaufman 2011] Kaufman, S., et al. Leakage in data mining. In: C. Apte, et al., Eds., ed. Proceedings of the 17th ACM SIGKDD international conference on Knowledge discovery and data mining - KDD '11, New York, New York, USA: ACM Press, 556.

[Kohavi 1995] Kohavi, R. A Study of Cross-Validation and Bootstrap for Accuracy Estimation and Model Selection, In International Joint Conference on Artificial Intelligence. In: International Joint Conference on Artificial Intelligence (IJCAI), 1995.

[Kohn 2011] Kohn, W., and Öztürk, R. Statistik für Ökonomen. Datenanalyse mit R und SPSS, Berlin, Heidelberg: Springer Berlin Heidelberg, 2011, 978-3642-14584-1.

[Kuhn 2013] Kuhn, M., and Johnson Kjells. Applied Predictive Modeling, New York: Springer New York, 2013.
[Kuhrke 2011] Kuhrke, B. Methode zur Energie- und Medienbedarfsbewertung spanender Werkzeugmaschinen. Dissertation, TU Darmstadt, 2011.

[Müller 2017] Müller, A., and Guido, S. Introduction to Machine Learning with Python - A Guide for Data Scientists, Beijing: O'Reilly Media, 2017.

[Pedregosa 2011] Pedregosa, F., et al. Scikit-learn: Machine learning in Python. Journal of machine learning research, 2011, 12, 2825-2830.

[Petruschke 2020] Petruschke, L., et al. Method to identify energy efficiency potentials of metal cutting machine tools in industry. Procedia CIRP, 2020, 90, 522-527, 10.1016/j.procir.2020.01.066.

[Petruschke 2021] Petruschke, L., et al. Machine learning based identification of energy states of metal cutting machine tools using load profiles. Paper accepted for publication. Procedia CIRP, 2021, 54th CIRP Conference on Manufacturing Systems.

[Posselt 2016] Posselt, G. Towards Energy Transparent Factories, Cham: Springer International Publishing, 2016, 978-3-319-20868-8.

[Sorrell 2000] Sorrell, S., et al. Reducing barriers to energy efficiency in private and public organisations. JOS3CT970022. Final Report., 2000.

http://publica.fraunhofer.de/eprints/urn_nbn_de_0011n-56745.pdf. Accessed 10 May 2021.

[Tewari 2005] Tewari, A., and Bartlett, P.L. On the Consistency of Multiclass Classification Methods. In: D. Hutchison, et al., Eds., ed. Learning Theory, Berlin, Heidelberg: Springer Berlin Heidelberg, 2005, 143-157.

[Umweltbundesamt 2021] Umweltbundesamt. Stromverbrauch: Entwicklung des Stromverbrauchs nach Sektoren, 2021.

https://www.umweltbundesamt.de/daten/energie/stromv erbrauch. Accessed 3 May 2021.

[VDI 1998] VDI. VDI 2225 Blatt 3:1998-11. Verein Deutscher Ingenieure e.V. - Konstruktionsmethodik Technisch-wirtschaftliches Konstruieren - Technisch wirtschaftliche Bewertung, Berlin: Beuth Verlag $\mathrm{GmbH}$, 1998.

[VDMA 2010] VDMA. VDMA Einheitsblatt 66412-1: Manufacturing Execution Systems (MES) Kennzahlen. Verband Deutscher Maschinen- und Anlagenbau e.V., Berlin: Beuth Verlag GmbH, 2010.

[VDMA 2019] VDMA. Messvorschrift zur Bestimmung des Energie- und Medienbedarfs von Werkzeugmaschinen in der Serienfertigung. Verband Deutscher Maschinenund Anlagenbau e.V., 2019, 25.080.01, 34179.

[Virtanen 2020] Virtanen, P., et al. SciPy 1.0: Fundamental Algorithms for Scientific Computing in Python. Nature Methods, 2020, 17, 261-272, 10.1038/s41592-0190686-2.

[Wirth 1998] Wirth, R., and Hipp, J. CRISP-DM: Towards a standard process model for data mining. In: AAAI Press and Editor: Rakesh Agrawal, Paul Stolorz, Eds., ed. Proceedings of the Fourth International Conference on the Practical Application of Knowledge Discovery and Data Mining, 29-39.

[Yang 2019] Yang, X.-S., et al. Third International Congress on Information and Communication Technology 797, Singapore: Springer Singapore, 2019, 978-981-13-1164-2. 